Supplement of Atmos. Meas. Tech., 12, 1717-1737, 2019

https://doi.org/10.5194/amt-12-1717-2019-supplement

(C) Author(s) 2019. This work is distributed under

the Creative Commons Attribution 4.0 License.

(c) (1)

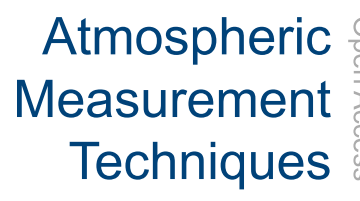

Supplement of

\title{
Marine liquid cloud geometric thickness retrieved from OCO-2's oxygen A-band spectrometer
}

Mark Richardson et al.

Correspondence to: Mark Richardson (markr@jpl.caltech.edu)

The copyright of individual parts of the supplement might differ from the CC BY 4.0 License. 


\section{S1 Relationships between optical and microphysical properties in subadiabatic boundary layer clouds}

\section{S1.1 Linking cloud microphysical and optical properties}

Here we present several key relationships summarised by Grosvenor et al. (2018), which resulted in relationships between cloud optical depth $\tau$, effective radius $r_{e}$, liquid water path $L W P$ and geometric thickness $H$ for subadiabatic clouds.

5 The extinction coefficient for a collection of droplets is:

$\beta_{\text {ext }}(z)=\pi \int_{0}^{\infty} Q_{\text {ext }}(r) r^{2} n(r) d r$

Where $Q_{e x t}$ is the extinction efficiency, the ratio of extinction and geometric cross section for a given droplet. Given that for cloud droplets in the A-band, $r_{e} \gg \lambda$, we take the approximation $Q_{e x t}=2$, which is the asymptotic limit from Mie theory for large size parameters.

10 Next we require the droplet effective radius, which is the ratio of the third to the second moments of the droplet size distribution:

$r_{e}(z)=\frac{\int_{0}^{\infty} r^{3} n(r) d r}{\left.\int_{0}^{\infty} r^{2} n(r) d r\right)}$

And the relation of liquid water content to the droplet volume:

$L(z)=\frac{4 \pi \rho_{w}}{3} \int_{0}^{\infty} r^{3} n(r) d r$

15 Combining Eqs. (S1) - (S3) results in:

$\beta_{\text {ext }}(z)=\frac{3 Q_{e x t}}{4 \rho_{w}} \frac{L(z)}{r_{e}(z)}$

We then introduce the ratio of the effective and volumetric mean droplet radii as:

$k=\left(\frac{r_{v}}{r_{e}}\right)^{3}$

For the commonly used gamma distribution of drop sizes, this is related to the width of the distribution. We assume that this

20 is constant with height, and its value tends to be near 0.8 in marine clouds. The liquid water content is also related to the volumetric mean radius via:

$L(z)=\frac{4 \pi \rho_{w} N_{d} r_{v}^{3}}{3}$

Combining Eqs. (S5) and (S6) and substituting into Eq. (S4), then integrating over the cloud gives optical depth:

$\tau_{c}=Q_{\text {ext }}\left(\frac{3}{4 \rho_{w}}\right)^{\frac{2}{3}}\left(N_{d} \pi k\right)^{\frac{1}{3}} \int_{z_{\text {base }}}^{z_{\text {top }}} L(z)^{\frac{2}{3}} d z$

\section{S1.2 Comparing subadiabatic and homogeneous clouds}

In the adiabatic cloud model we have:

$L(z)=f_{a d} c_{w} Z$ 
And therefore, taking geometric thickness $H$ as $z_{\text {top }}$-zbase:

$\tau_{c, a d}=\frac{3 Q_{e x t}}{5}\left(\frac{3 f_{a d} c_{w}}{4 \rho_{w}}\right)^{\frac{2}{3}}\left(N_{d} \pi k\right)^{\frac{1}{3}} H^{\frac{5}{3}}$

For a homogeneous cloud we have a constant liquid water content with height, i.e. $L(z)=A$ :

$\tau_{c, h o m o}=Q_{\text {ext }}\left(\frac{3 A}{4 \rho_{w}}\right)^{\frac{2}{3}}\left(N_{d} \pi k\right)^{\frac{1}{3}} H$

5 The homogeneous cloud optical depth differs from the subadiabatic cloud in that optical depth scales with $H$ instead of $H^{5 / 3}$, and otherwise differs by a ratio of $5 A^{2 / 3} / 3$. We can compare their optical properties by consider the effect on optical depth of the difference in structure for an equal $L W P$. In this case, to conserve mass:

$A=\frac{f_{a d} c_{w} H}{2}$

And Eq. (S10) becomes:

$10 \tau_{c, h o m o}=Q_{\text {ext }}\left(\frac{3 f_{a d} c_{w}}{8 \rho_{w}}\right)^{\frac{2}{3}}\left(N_{d} \pi k\right)^{\frac{1}{3}} H^{\frac{5}{3}}$

Or more explicitly we compare $\tau_{c, a d}$ for the subadiabatic cloud and $\tau_{c, h o m o}$ for the homogeneous cloud first by simplifying:

$X=Q_{\text {ext }}\left(\frac{3 f_{a d} c_{w}}{4 \rho_{w}}\right)^{\frac{2}{3}}(\pi k)^{\frac{1}{3}}$

Which makes the optical depths:

$\tau_{c, a d}=\frac{3}{5} X N_{d, a d}^{\frac{1}{3}} H^{\frac{5}{3}}$

15 And:

$\tau_{c, h o m o}=2^{-\frac{2}{3}} X N_{d, h o m o}^{\frac{1}{3}} H^{\frac{5}{3}}$

i.e. these two clouds show the same scaling of $\tau_{c}$ with $H$ but vary by a factor of:

$\frac{\tau_{c, h o m o}}{\tau_{c, a d}}=\frac{5}{3} 2^{-\frac{2}{3}}\left(\frac{N_{d, h o m o}}{N_{d, a d}}\right)^{\frac{1}{3}}$

Note that while $N_{d}$ is constant within each cloud, it is not necessarily the same in the homogeneous case as it is in the

20 subadiabatic case.

Referring back to Equations (S3) and (S5) and $N_{d}$ constant with height:

$L(z)=\frac{4 \pi \rho N_{d} r_{e}^{3}(z)}{3 k}$

With the subadiabatic $L(z)$ this can be rearranged to determine that $N_{d, a d}^{\frac{1}{3}}$ is:

$N_{d, a d}^{\frac{1}{3}}=\left(\frac{3 k f_{a d} c_{w}}{4 \pi \rho_{w}}\right)^{\frac{1}{3}} z^{\frac{1}{3}} r_{e, a d}^{-1}(z)$

25 Whereas with the homogeneous $L(z)$ this becomes:

$N_{d, \text { homo }}^{\frac{1}{3}}=\left(\frac{3 k f_{a d} c_{w}}{4 \pi \rho_{w}}\right)^{\frac{1}{3}}\left(\frac{H}{2}\right)^{\frac{1}{3}} r_{e, \text { homo }}^{-1}$ 
When substituting Eqs. (S18) and (S19) into (S16), the first terms in the brackets cancel and we obtain:

$\frac{\tau_{c, h o m o}}{\tau_{c, a d}}=\frac{5}{3} 2^{-\frac{2}{3}}\left(\frac{H}{2 z}\right)^{\frac{1}{3}} \frac{r_{e, a d}(z)}{r_{e, h o m o}}=\frac{5}{6}\left(\frac{H}{z}\right)^{\frac{1}{3}} \frac{r_{e, a d}(z)}{r_{e, h o m o}}$

Therefore a homogeneous cloud has the same optical depth as a subadiabatic cloud of the same $H$ and $L W P$ provided that:

$r_{e, \text { homo }}=\frac{5}{6} r_{e, a d}(H)$

5 Or alternatively:

$r_{e, \text { homo }}=r_{e, a d}\left(\frac{125}{216} H\right) \cong r_{e, a d}(0.58 H)$

\section{S1.3 Calculating prior cloud thickness}

The subadiabatic and homogeneous clouds are expected to have the same optical depth for a fixed $H$ and $L W P$ given that an appropriate $r_{e}$ is used. The next question is how to calculate an appropriate prior cloud pressure thickness. As part of the

10 OCO2CLD-LIDAR-AUX retrieval, we make a prior estimate of the cloud optical depth based on a lookup table using the continuum A-band radiances. And the retrieval assumed $r_{e}=12 \mu \mathrm{m}$. Therefore we express $L W P$ as $L W P\left(\tau, r_{e}\right)$ and relate this to $H$ using Eq. (S8). Firstly, we combine Eq. (S8) and (S17) and evaluate them at cloud top:

$L(H)=f_{a d} c_{w} H=\frac{4 \pi \rho_{w} N_{d} k r_{e}^{3}}{3}$

$H=\frac{4 \pi \rho_{w} N_{d} k r_{e}^{3}}{3 f_{a d} c_{w}}$

15 Integrating Eq. (S8) and inserting for $H$ using (S24):

$L W P=\frac{1}{2} f_{a d} c_{w} H^{2}=\frac{1}{2} f_{a d} c_{w}\left(\frac{4 \pi \rho_{w} N_{d} k}{3 k f_{a d} c_{w}}\right)^{2} r_{e}^{6}$

Next, we also insert Eq. (S24)'s expression for $H$ into Eq. (S9), for the adiabatic $\tau_{c}(H)$ :

$\tau_{c}=\frac{3 Q_{e x t}}{5}\left(\frac{3 f_{a d} c_{w}}{4 \rho_{w}}\right)^{\frac{2}{3}}\left(N_{d} \pi k\right)^{\frac{1}{3}}\left(\frac{4 \pi \rho_{w} N_{d} k r_{e}^{3}}{3 f_{a d} c_{w}}\right)^{\frac{5}{3}}$

Collecting the terms in Eq. (S26) and expressing as a function of $r_{e}^{5}$ :

$20 r_{e}^{5}=\frac{5 f_{a d} c_{w}}{4 Q_{e x t} \rho_{w}}\left(N_{d} \pi k\right)^{-2} \tau$

Inserting this into Eq. (S2):

$L W P=\frac{1}{2} f_{a d} c_{w}\left(\frac{4 \pi \rho_{w} N_{d} k}{3 f_{a d} c_{w}}\right)^{2} \frac{5 f_{a d} c_{w}}{4 Q_{e x t} \rho_{w}}\left(N_{d} \pi k\right)^{-2} r_{e} \tau$

Once again, collecting and cancelling terms results in:

$L W P=\frac{10 \rho_{w}}{9 Q_{e x t}} r_{e} \tau$

25 Or for $H$,

$H=\sqrt{\frac{20 \rho_{w} r_{e} \tau}{9 Q_{e x t} f_{a d} c_{w}}}$ 
We take the following values: $f_{a d} c_{w}=1.9 \times 10^{-3} \mathrm{~g} \mathrm{~m}^{-4}, Q_{e x t}=2$ and $\rho_{w}=1,000 \mathrm{~kg} \mathrm{~m}^{-3}$. Note that $r_{e}$ here refers to the cloud top value. From Eq. (S21), the homogeneous value to use is equivalent to the $5 / 6$ ths of this value.

\section{Vertical profile of extinction in subadiabatic and homogeneous clouds}

Taking Eq. (S3), which relates extinction to $L(z)$ and $r_{e}(z)$, we consider their vertical profiles in a subadiabatic cloud, rearrange 5 Eq. (S6) and use Eq. (S5) to make $r_{e}(z)$ the subject:

$r_{e}(z)=\left(\frac{3 L(z)}{4 \pi \rho_{w} N_{d} k}\right)^{\frac{1}{3}}$

Substitute this into Eq. S4:

$\beta_{\text {ext }}(z)=\frac{3 Q_{\text {ext }}}{4 \rho_{w}}\left(\frac{4 \pi \rho_{w N_{d} k}}{3}\right)^{\frac{1}{3}} L^{\frac{2}{3}}(z)$

And taking the liquid water profile from Eq. (S8):

$10 \beta_{\text {ext }}(z)=\frac{3 Q_{e x t}}{4 \rho_{w}}\left(\frac{4 \pi \rho_{w} N_{d} k}{3}\right)^{\frac{1}{3}}\left(f_{a d} c_{w} z\right)^{\frac{2}{3}}$

i.e. the extinction scales with $z^{2 / 3}$ in a subadiabatic cloud and:

$\beta_{\text {ext }}(z)=A z^{\frac{2}{3}}$

Integrating Eq. (S35) shows that $A$ is related to $H$ and $\tau$ and:

$\beta_{\text {ext }}(z)=\frac{5 \tau}{3 H^{\frac{5}{3}}} z^{\frac{2}{3}}$

15 This contrasts with a homogeneous cloud whose profile is:

$\beta_{\text {ext }}(z)=\frac{\tau}{H}$

For constant $H$ and $\tau$, example extinction profiles are displayed in Figure S1. It is clear that the cloud extinction in the subadiabatic model is weighted more heavily towards cloud top. 


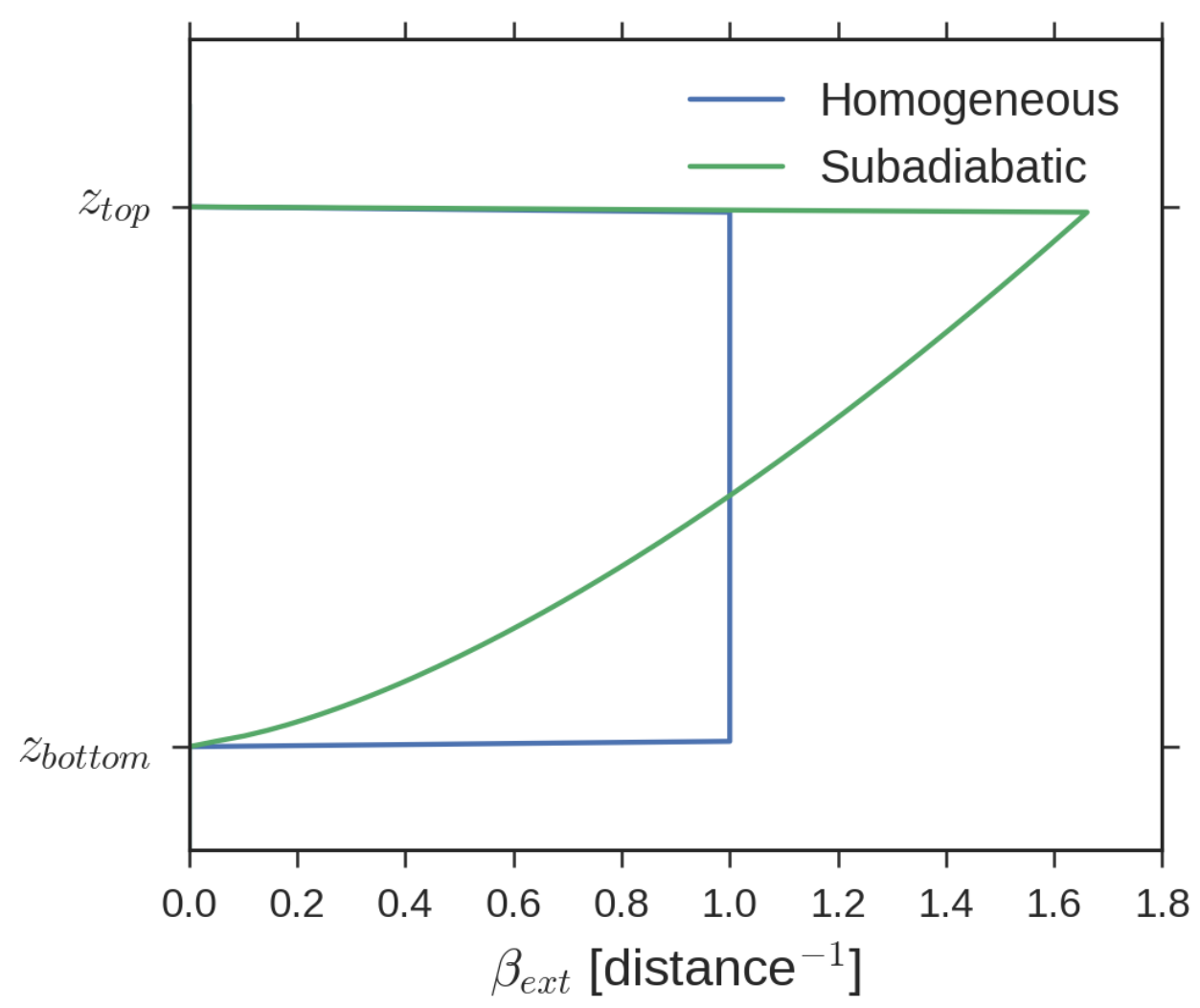

Figure S1. Example extinction coefficients for a cloud of the same $H$ and $\tau$ for a homogeneous or subadiabatic structure. This ignores atmospheric molecular absorption. 